\title{
On the Necessary and Sufficient Assumptions for UC Computation*
}

\author{
Ivan Damgård, Jesper Buus Nielsen, and Claudio Orlandi \\ Department of Computer Science, Aarhus University \\ \{ivan, jbn, claudio\}@cs.au.dk
}

\begin{abstract}
We study the necessary and sufficient assumptions for universally composable (UC) computation, both in terms of setup and computational assumptions. We look at the common reference string model, the uniform random string model and the key-registration authority model (KRA), and provide new results for all of them. Perhaps most interestingly we show that:

- For even the minimal meaningful KRA, where we only assume that the secret key is a value which is hard to compute from the public key, one can UC securely compute any poly-time functionality if there exists a passive secure oblivious-transfer protocol for the stand-alone model. Since a KRA where the secret keys can be computed from the public keys is useless, and some setup assumption is needed for UC secure computation, this establishes the best we could hope for the KRA model: any non-trivial KRA is sufficient for UC computation.

- We show that in the KRA model one-way functions are sufficient for UC commitment and UC zero-knowledge. These are the first examples of UC secure protocols for non-trivial tasks which do not assume the existence of public-key primitives. In particular, the protocols show that non-trivial UC computation is possible in Minicrypt.
\end{abstract}

\section{Introduction}

We study the necessary and sufficient assumptions for universally composable (UC) computation [CanU1, both in terms of which setup models are needed and how strong assumptions on the setup are needed, and in terms of necessary and sufficient computational assumptions.

One of the motivation is to study the minimal setup required for UC computation. It is known that some kind of setup is required, which makes it a theoretically interesting question exactly how strong an assumption must be made on the setup. We study both the common reference string model (CRS) and the key registration authority model (KRA), and some variations.

The goal of the study is also to determine the relationships between one way functions (OWF), passive secure stand-alone oblivious transfer (SA-OT)

The original version of this chapter was revised: The copyright line was incorrect. This has been corrected. The Erratum to this chapter is available at DOI: 10.1007/978-3-642-11799-2_36

* A full version of this work can be found at http://eprint.iacr.org/2009/247

${ }^{1}$ If a passive secure SA-OT exists then also an active secure SA-OT exists via standard compilation techniques.

D. Micciancio (Ed.): TCC 2010, LNCS 5978, pp. 109 127, 2010.

(C) Springer-Verlag Berlin Heidelberg 2010 
commitments (UC-Com) and UC oblivious transfer (UC-OT) in different set-up model.2: for stand-alone security, we know that OWF are sufficient for other cryptographic tasks such as commitments and zero-knowledge proofs. Are OWF equivalent to any of these tasks when it comes to UC security? For the CRS models, Damgård and Groth DG03 answered the question negatively showing that UC-Com implies key agreement in the CRS models and therefore, given the black-box separation between OWF and key agreement IR89, RTV04, OWF are not sufficient to realize UC-Com. We find it interesting to study if this is inherent or associated to the particular setup model. We include SA-OT because it is complete for stand alone computation Kil88, and therefore a natural question is whether SA-OT is sufficient also for UC computation. On the other hand it is interesting to know whether this assumption is minimal, i.e. whether SA-OT is also necessary to implement UC-Com and UC-OT. The motivation for including UC-OT is that it is complete for general UC computation: it is possible to implement any well-formed ideal functionality given the UC-OT functionality, see CLOS02, IPS08. Finally the motivation for including UC-Com is that it is potentially weaker than UC-OT but still implies a number of non-trivial tasks like coin-flip and zero-knowledge.

Highlights. We highlight some of the new findings which we find particularly interesting: In the KRA model, we provide the first construction of UC commitment from one-way functions - all previous constructions, to the best of our knowledge, used special assumptions or assumed at least public-key encryption. A consequence of it is that zero-knowledge and coin-flip can be UC securely implemented in Minicrypt. Until now it was not known if any non-trivial UC computation was possible in Minicrypt.

Remembering that UC-Com implies SA-OT in the CRS models we get another new result: The choice of the setup model can make a difference in which ideal functionalities can be implemented under a given computational assumption. In the CRS model we need SA-OT for UC-Com, but in the KRA model we can do with just OWF. This seems to be the first such separation of the setup models.

It turns out that SA-OT is sufficient for UC-OT in any setup model we considered, in particular in the minimal version of both the CRS and KRA model. Since some setup assumption is needed for general UC computation, this seems a very positive addition to the UC theory: Some setup is needed, but even the most trivial setup will allow to implement any well-formed functionality.

Finally, we show how to implement authenticated channels given a minimal meaningful KRA: Implementing authentication in a public-key setting is of course trivial if one can choose the structure of the public keys - one includes a verification key in the public key and signs all messages. It is by far trivial in our relaxed KRA model as we make no assumption on the public keys except that they are in the range of some one-way function, which might itself be maliciously chosen. Standard constructions of signature schemes from one-way functions use verification keys with much more structure than this. This seems

\footnotetext{
${ }^{2}$ When writing UC-Com or UC-OT we mean the multi-party, multi-session version of the protocols.
} 
to be the first result which shows that any value you could hope for to act as a public key can actually be used to implement an authenticated channel.

Setup models: We look at five setup models:

- In the uniform common random string ( $U-C R S$ ) model we assume that a single uniformly random $\ell$-bit string crs is chosen by a trusted party and made public. Here $\ell$ is chosen by the protocol.

- In the chosen common reference string $(C$-CRS) model the trusted party samples crs using a poly-time one-way $3^{3}$ distribution $D:\{0,1\}^{\kappa} \rightarrow\{0,1\}^{\ell}$, which allows crs to have a particular form. We assume that the trusted party samples a single $c r s=D(r)$ for uniformly random $r \in\{0,1\}^{\kappa}$ and makes crs public. The function $D$ might be given by the protocol $\pi$.

- The any common reference string $(A-C R S)$ model is like the C-CRS model, except that we let the adversary pick $D$, under the only restriction that $D$ is one-way. The trusted party samples $c r s=D(r)$ and makes $(D, c r s)$ public. 4

- In the chosen key registration authority ( $C$-KRA) model we assume that the protocol contains a poly-time function $f_{i}$ for each party $P_{i}$. A trusted party will sample $p k_{i}=f_{i}\left(s_{i}\right)$ for each $P_{i}$ and give $s_{i}$ to $P_{i}$ and $p k_{i}$ to all other parties. This models a key-registration authority with public keys $p k_{i}$, secret keys $s_{i}$ and where the parties are guaranteed to know their secret keys. 5

- The any key registration authority ( $A$-KRA) model is like the C-KRA model, except that we allow the adversary to specify each $f_{i}$, under the only restriction that $f_{i}$ is a one-way function when $P_{i}$ is honest.

In the CRS models we in addition assume the presence of authenticated channels, as the existence of a CRS clearly does not allow authentication: All parties know the CRS and nothing else, so nothing distinguishes an honest party from the adversary. In the KRA models we start from the unauthenticated channels model, as the existence of a public-key infrastructure has the potential to allow authentication. In the A-CRS model the protocol $\pi$ does not choose $D$, and the security of $\pi$ should hold for any one-way function $D$. Another way of phrasing the model is to say that the protocol $\pi$, parametrized by $D$, should be secure in the C-CRS model for any one-way function $D$. In some sense this models the minimal meaningful common random string: we do not make assumptions on how random it is, but the parties can agree on the fact that there is something about the string which neither of them knows.

The A-KRA model in some sense is the minimal meaningful assumption on a key registration authority: Each party has a publicly known public key $p k_{i}$ and

${ }^{3}$ If $D$ is invertible then the C-CRS and the U-CRS model are trivially equivalent.

${ }^{4}$ Note that the A-CRS model generalizes both the U-CRS and the C-CRS, for computational security: the U-CRS is computationally indistinguishable from a setup where the trusted party picks a random seed and expands it using a pseudo random generator.

${ }^{5}$ This is essentially the key registration with knowledge (KRK) model from BCNP04. We cannot start from the KR model from [BCNP04] as we need that parties know their secret keys to implemented authenticated channels. 
there is some secret about $p k_{i}$ which only $P_{i}$ knows. A protocol for the A-KRA can therefore be run given any meaningful key registration authority, with no assumption whatsoever about the form of $p k_{i}$ or the exact hardness of finding $s_{i}$. Note that one can think of simpler public key models, as the bare public key model (BPK) introduced in CGGM00, that does not require corrupted parties to know their secret keys. However, even if the BPK model has been successfully used to break some impossibility results about concurrent ZK (see OPV08 and reference therein), it was shown in [KL07 that UC computation is impossible even in the BPK model or any other public key model "without knowledge".

Results: Let $C_{1}, C_{2} \in\{\mathrm{OWF}, \mathrm{SA}-\mathrm{OT}, \mathrm{UC}-\mathrm{Com}, \mathrm{UC}-\mathrm{OT}\}$ and $M$ one of the described setup model. Then we can ask ourselves questions of the form "is $C_{1}$ necessary/sufficient for $C_{2}$ in the $M$ model?" Some of these are of course trivial, like is OWF necessary for $U C$-OT in the $U$-CRS model?, but many are non-trivial and theoretically interesting, like is SA-OT sufficient for UC-OT in the A-CRS model?. The answers to these questions are pictorially illustrated in Figures [1] and 2] The main results are proved through the text while the implications that were already known, or that easily follow from our results and known facts are presented in Sec. 8]

When SA-OT appears on the left side of the implication, the assumption is that there exist a protocol that implement SA-OT in the stand alone mode. When UC-Com and UC-OT appear on the left side of the implication, the assumption is that there exist a protocol that implement that functionality in the model in question, i.e., we are not referring to an hybrid world where the functionality is given to the party: We assume that the parties know a protocol to implement the functionality.

Note that the figure states that UC-Com is not sufficient for UC-OT in the C-KRA model, while the answer is yes in the A-KRA model. This may seem surprising because the C-KRA model (unlike A-KRA) allows the protocol to

$\frac{\mathrm{U} / \mathrm{C} / \mathrm{A}-\mathrm{CRS}, \mathrm{C} / \mathrm{A}-\mathrm{KRA}}{\mathrm{OWF}^{\sharp} \Uparrow_{\mathrm{UC}-\mathrm{COM}}^{\Uparrow} \mathbb{\mathrm { UT }}^{\mathrm{SA}-\mathrm{OT}}}$

Fig. 1. One direction of the relationship between the primitives holds in any setup model

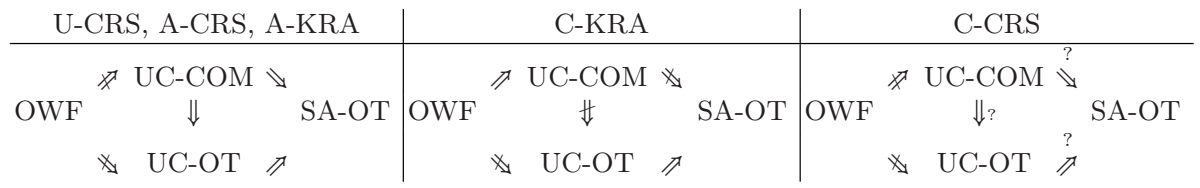

Fig. 2. The other direction differs in different setup models 
choose how public keys are computed and so it seems that anything that is possible in the A-KRA model should also possible in C-KRA. The catch is that the UC-Com assumption is not the same in the two models, in particular, having a UC-commitment scheme that works in the A-KRA model is a much stronger tool than one that needs C-KRA.

For all our negative answers to sufficient questions and positive answers to necessary questions, we mean that there is no black-box construction. We cannot answer whether OWF is sufficient for UC-Com with our current understanding of complexity theory: It might be that one-way functions do not exists, in which case the assumption $\mathrm{OWF}$ is false, and then $\mathrm{OWF} \Rightarrow \mathrm{UC}-\mathrm{Com}$ is true. The result $\mathrm{UC}-\mathrm{Com} \stackrel{(\mathrm{C}-\mathrm{KRA})}{\Rightarrow} \mathrm{UC}-\mathrm{OT}$ therefore means that there is no black-box construction which takes an implementation of UC commitment for the C-KRA model and gives an implementation of UC OT for the C-KRA model. For some of our positive answers to sufficient questions and negative answers to necessary questions, we appeal to non-black box constructions. As an example, the result $\mathrm{OWF} \stackrel{(\mathrm{C}-\mathrm{KRA})}{\Rightarrow} \mathrm{UC}-$ Com uses a description of the circuit for the OWF 6

Related work. In CLOS02 the main feasibility result for UC computation in the CRS model can be found. CLOS02 needs to assume enhanced trapdoor permutation in order to achieve their results, while we use the strictly weaker assumption SA-OT. On the other hand this comparison is not quite fair as CLOS02 tries to achieve adaptive security, and consider static security just as a special case, while our focus is entirely on static security. In [LPV09 a general framework for UC feasibility results is presented, showing how different setup assumptions (including timing model, tamper proof hardware, etc.) can be seen as different implementations of what the authors call UC puzzles. While in LPV09 the results are proved assuming the existence of enhanced trapdoor permutation, we look at strictly weaker assumptions as OWF and SA-OT.

In a recent series of papers PR08, IMPR09, the classification of the cryptographic complexity of UC functionalities is studied. Perhaps most interestingly with respect to our work, in [MPR09 it is shown the SA-OT assumption is equivalent to any UC functionality being either trivial or complete. There is a clear overlap between these results and some of ours, however we focus on setup functionalities - that are invoked just once at the beginning of the protocol, while the constructions in MPR09 use the ideal functionalities during the protocol in an on-line fashion.

\section{The KRA Model}

In this section we give our model of minimal public-key setup, where each party knows a secret which is not known by the other parties. We associate these

${ }^{6}$ It might be possible that $A \nRightarrow B$ and $A \Rightarrow B$ at the same time. However, if for any of the $\nRightarrow$ separations in Figure [2] this is the case, then one would have a non-black-box construction of SA-OT using OWF. Such a construction is unlikely to exist - or at least requires completely new cryptographic techniques - see also [RTV04. 
secrets to public values which we distribute via a key generator $G$. When sampled it outputs $\left(\left(R_{1}, s_{1}\right), \ldots,\left(R_{n}, s_{n}\right)\right)$, where each $R_{i}$ is a description of a PPT set and $s_{i}$ is the secret of $P_{i}$, and $s_{i} \in R_{i}$ for $i \in[n]=\{1, \ldots, n\}$. We call $R=\left(R_{1}, R_{2}, \ldots, R_{n}\right)$. For a "normal" KRA we would have that the description of $R$ contains the parties' public keys $p k_{1}, \ldots, p k_{n}$ and that $s_{i} \in R_{i}$ if $s_{i}$ is the secret key associated to $p k_{i}$; in this case we will write $\left(p k_{i}, s_{i}\right) \in R_{i}$. To model that a party's secret $s_{i}$ is hard to find for the other parties we require that it is hard to find any $s_{i}^{\prime}$ such that $s_{i}^{\prime} \in R_{i}$. This should hold even if one is given $R \cup\left\{s_{j}\right\}_{j \in[n] \backslash\{i\}}$.

To allow corrupted parties to use secrets different from those of the honest parties (maybe fixed instead of random or even of another form), we let $G$ depend on the set $C$ of corrupted parties, and we let the adversary $\mathcal{A}$ influence the key generation as follows: Both $G$ and $\mathcal{A}$ are ITMs. First $G$ is given input $n$ and $C$, where $n$ defines the number of parties and $C \subset[n]$ defines the set of corrupted parties. Then $G$ and $\mathcal{A}$ interact and at some point $G$ outputs $\left(R, s_{1}, \ldots, s_{n}\right)$; we write $\left(R, s_{1}, \ldots, s_{n}\right) \leftarrow(G(n, C), \mathcal{A})$. For $G$ to be meaningful we require that $s_{j} \in R_{j}$ for all parties $P_{j \in[n]}$. We only require that the secrets of honest parties, $P_{i \in[n] \backslash C}$, are hard to find, as it is not necessarily meaningful to require that corrupted parties keep their secrets hidden.

We introduce some convenient notation for the case where all public keys are generated using the same function $f$. For a function $f:\{0,1\}^{\kappa} \rightarrow\{0,1\}^{\ell}$ we define the key generator $G^{f}$ as follows: For each honest party $P_{i}$ it samples $s_{i} \in\{0,1\}^{\kappa}$ and computes $p k_{i}=f\left(s_{i}\right)$. Then it outputs $\left\{p k_{i}\right\}_{i \in[n] \backslash C}$ to the adviser. It interprets the next message from the adviser as a set $\left\{s_{i}\right\}_{i \in C}$ and computes $p k_{i}=f\left(s_{i}\right)$ for $i \in C$. It defines $R^{f}$ by $(p k, s) \in R^{f}$ iff $p k=f(s)$ and then outputs $\left(\left(p k_{1}, s_{1}, R^{f}\right), \ldots,\left(p k_{n}, s_{n}, R^{f}\right)\right)$.

- Let $n$ be the number of parties and $C$ the set of corrupted parties, and run $G(n, C)$ with the UC adversary $\mathcal{A}$ as adviser.

- When $G(n, C)$ outputs $\left(R, s_{1}, \ldots, s_{n}\right)$, then send $\left(R,\left\{s_{i}\right\}_{i \in C}\right)$ to $\mathcal{A}$ and send $\left(R, s_{i}\right)$ to each $P_{i}$, letting $\mathcal{A}$ determine the delivery time.

Fig. 3. The KRA ideal functionality $\mathcal{F}_{\text {KRA }}^{G}$ for a generator $G$

Definition 1. We call $G$ meaningful if $\forall \mathcal{A}, \mathcal{A}$ wins the following game with negligible probability: Run $\mathcal{A}$ to get $(n, C)$, with $n$ polynomially bounded and $C \subset[n]$. Then sample $\left(R, s_{1}, \ldots, s_{n}\right) \leftarrow(G(n, C), \mathcal{A})$. At this point $\mathcal{A}$ wins if $\exists j \in[n] s_{j} \notin R_{j}$. If $\mathcal{A}$ did not win here, run $\mathcal{A}$ on $R$ to get $i \in[n] \backslash C$, and run $\mathcal{A}$ on $s_{-i}=\left\{s_{j}\right\}_{j \in[n] \backslash\{i\}}$ to get an output $\left(i, s_{i}^{\prime}\right)$. If $s_{i}^{\prime} \in R_{i}$, then $\mathcal{A}$ wins.

Definition 2. Let $\mathcal{G}$ be a set of key generators, let $\pi$ be a protocol and $\mathcal{F}$ an ideal functionality. We say that $\pi$ is a UC secure implementation of $\mathcal{F}$ with a $\mathcal{G} K R A$ if $\pi$ is a UC secure implementation of $\mathcal{F}$ in the $\mathcal{F}_{\mathrm{KRA}}^{G}$-hybrid model (Fig. (3) for all $G \in \mathcal{G}$. We say that $\pi$ is a UC secure implementation of $\mathcal{F}$ with any meaningful KRA (A-KRA) if the above holds for $\mathcal{G}$ being the set of all meaningful key generators. 


\section{Authentication in the A-KRA Model given OWF}

We show here how to implement authentication with any meaningful KRA. We first construct a system for identification secure under concurrent composition, using $\Sigma$-protocols in a more or less standard manner. Then we extend this identification system to a UC secure authentication system in a novel manner.

Implementing authentication in a public-key setting is of course trivial if one can choose the structure of the public keys - one includes a verification key in the public key and signs all messages. It is by far trivial in our relaxed KRA model as we make no assumption on the public keys except that they are in the range of some one-way function, which might itself be maliciously chosen. Standard constructions of signature schemes from one-way functions use verification keys with much more structure than this.

\section{$3.1 \quad \Sigma$-Protocols}

For details on the following brief introduction see CDS94. Let $R \subseteq\{0,1\}^{*} \times$ $\{0,1\}^{*}$ be a binary relation. A $\Sigma$-protocol for $R$ consists of $(A, E, Z, J, W, S)$, where $A$ is a poly-time algorithm which for all $(x, w) \in R$ and sufficiently long randomness $r$ outputs a commit message $a=A(x, w, r) ; E=\{0,1\}^{\ell}$ is a set of challenges; $Z$ is a poly-time algorithm which given $(x, w) \in R$ and $e \in E$ and randomness $r$ outputs a reply $z=Z(x, w, e, r) ; J$ is a poly-time algorithm, called the judgment, which given any $(x, a, e, z)$ outputs $J(x, a, e, z) \in\{$ accept, reject $\}$; and $W$ is a poly-time algorithm called the witness extractor and $S$ is a PPT algorithm called the simulator. Furthermore:

completeness: For all $(x, w) \in R$, all randomness $r$ and $a=A(x, w, r)$ and $z=Z(x, w, e, r)$ it holds that $J(x, a, e, z)=$ accept.

special soundness: For all $(x, a, e, z)$ and $\left(x, a, e^{\prime}, z^{\prime}\right)$ with $e \neq e^{\prime}, V(x, a, e, z)=$ accept and $J\left(x, a, e^{\prime}, z^{\prime}\right)=$ accept it holds that $\left(x, W\left(x, a, e, z, e^{\prime}, z^{\prime}\right)\right) \in R$.

honest verifier zero-knowledge: For all $(x, w) \in R$ and all $e \in E$ the simulator outputs $(a, z) \leftarrow S(x, e)$ such that $J(x, a, e, z)=$ accept and such that the distribution of $(x, a, e, z)$ is computationally indistinguishable from $(x, A(x, w, r), e, Z(x, w, e, r))$ for a uniformly random $r$. This holds even when the distinguisher is given $w$.

One round of the standard zero-knowledge protocol for Hamiltonian Cycle using a statistically binding commitment scheme is a $\Sigma$-protocol for Hamiltonian Cycle with $E=\{0,1\}$. Since $\Sigma$-protocols are closed under parallel composition, this gives a $\Sigma$-protocol for any NP relation $R$ based on one-way function, with $E=$ $\{0,1\}^{\ell}$ for any polynomial $\ell$.

Let $R_{0}$ and $R_{1}$ be binary relations and define $R=R_{0} \vee R_{1}$ by $\left(\left(x_{0}, x_{1}\right), w\right) \in R$ iff $\left(x_{0}, w\right) \in R_{0}$ or $\left(x_{1}, w\right) \in R_{1}$. Then given two $\Sigma$-protocols $\Sigma_{0}, \Sigma_{1}$ for $R_{0}, R_{1}$ respectively, we can use the OR-construction to construct a $\Sigma$-protocol, $\Sigma=$ $\Sigma_{0} \vee \Sigma_{1}$ for the relation $R=R_{0} \vee R_{1}$. Let $x=\left(x_{0}, x_{1}\right)$ be an instance for which there exists $w_{0}$ and $w_{1}$ such that $\left(x_{0}, w_{0}\right) \in R_{0}$ and $\left(x_{1}, w_{1}\right) \in R_{1}$. Then the OR-construction is witness indistinguishable in the following sense: Consider a 
PPT adversary $\mathcal{A}$. Give it $\left(x,\left(w_{0}, w_{1}\right)\right)$ and give it access to a proof oracle $\mathcal{O}_{b}$, which on input prv picks a fresh identifier $I$, samples $a^{(I)} \leftarrow A\left(x, w_{b}, r\right)$, stores the prover intermediate state $P^{(I)}=(I, b, r)$ and returns $a^{(I)}$ to $\mathcal{A}$. On an input (chl, $\left.I, e^{(I)}\right)$ when some $P^{(I)}=(I, b, r)$ is stored, it deletes $P^{(I)}$, computes $z^{(I)}=Z\left(x, w_{b}, e^{(I)}, r\right)$ and returns $z^{(I)}$ to $\mathcal{A}$. At the end $\mathcal{A}$ outputs a guess at $b$. Then $\left|\operatorname{Pr}\left[\mathcal{A}^{\mathcal{O}_{0}}\left(x,\left(w_{0}, w_{1}\right)\right)=0\right]-\operatorname{Pr}\left[\mathcal{A}^{\mathcal{O}_{1}}\left(x,\left(w_{0}, w_{1}\right)\right)=0\right]\right|$ is negligible.

We call $G$ a hard double-witness generator for $R=R_{0} \vee R_{1}$ if it is PPT and a random sample $\left(x, w_{0}, w_{1}\right) \leftarrow G$ has the property that $\left(x, w_{0}\right) \in R$ and $\left(x, w_{1}\right) \in R$ and that it is hard to compute $w_{0}$ from $\left(x, w_{1}\right)$ and hard to compute $w_{1}$ from $\left(x, w_{0}\right)$, i.e., a PPT algorithm given a random $\left(x, w_{b}\right)$ outputs $\left(x, w_{1-b}\right)$ with negligible probability. If $G$ is a hard double-witness generator for $R=R_{0} \vee R_{1}$, then $\Sigma=\Sigma_{0} \vee \Sigma_{1}$ is witness hiding for $G$, i.e., an adversary cannot compute a witness after seeing any number of proofs. Since $\Sigma$-protocol are proofs of knowledge, the adversary cannot give a proof without knowing the witness. Putting these two observations together we get that the adversary cannot give a proof for a statement $x$ even after seeing any number of proofs for $x$, in the following sense: We say that $\mathcal{A}$ wins the reprove game in Fig. 4 if at the end of the game there is a stored value $(a, e, z)$ (from reply verifier), where $J(x, a, e, z)=$ accept and where $\mathcal{A}$ did not challenge a prover (in reply verifier) between receiving $e$ and returning $z$. I.e., $\mathcal{A}$ did not challenge a prover while it had to compute its own challenge.

initialize Let $I=0$. Sample $\left(x, w_{0}, w_{1}\right) \leftarrow G$ and give $x$ to $\mathcal{A}$.

start prover Whenever $\mathcal{A}$ inputs (prv, $b$ ), let $I=I+1$, sample $a^{(I)} \leftarrow A\left(x, w_{b}, r\right)$, store the prover intermediate state $P^{(I)}=(I, b, r)$ and return $a^{(I)}$ to $\mathcal{A}$.

challenge prover Whenever $\mathcal{A}$ inputs $\left(\mathrm{chl}, I, e^{(I)}\right)$ and some $P^{(I)}=(I, b, r)$ is stored, delete $P^{(I)}$, compute $z^{(I)}=Z\left(x, w_{b}, e^{(I)}, r\right)$ and return $z^{(I)}$ to $\mathcal{A}$.

start verifier On input (verify, $a$ ) from $\mathcal{A}$, sample a uniformly random $e \epsilon_{R} E$, store $(a, e)$ and return $e$ to $\mathcal{A}$.

reply verifier On input (reply, $a, e, z)$ from $\mathcal{A}$, where $(a, e)$ is stored, delete $(a, e)$ and store $(a, e, z)$.

Fig. 4. The reprove game for $\mathcal{A}, \Sigma$ and $G$

Theorem 1. Let $\Sigma_{0}$ be a $\Sigma$-protocol for $R_{0}, \Sigma_{1}$ be a $\Sigma$-protocol for $R_{1}$ and $G$ be a hard double-witness generator for $R=R_{0} \vee R_{1}$. Then for all $\mathcal{A}$ PPT verifiers, $\mathcal{A}$ wins the reprove game with $\Sigma=\Sigma_{0} \vee \Sigma_{1}$ and $G$ with negligible probability.

The intuition behind the proof is that an adversary $\mathcal{A}$ which wins the game can be used to extract a witness by rewinding the winning conversation and sending a new challenge, to get two valid conversations. Since $\mathcal{A}$ did not challenge a prover between getting $e$ and sending its reply, the rewinding does not give problems. Which of the two witnesses $w_{0}$ and $w_{1}$ is extracted by $\mathcal{A}$ does not change significantly if we give all the proofs to $\mathcal{A}$ using a random fixed witness $w_{b}$ instead of letting $\mathcal{A}$ choose $b$ from proof to proof: If it did, it would clearly allow 
us to break witness indistinguishability. So, with a non-negligible probability $\mathcal{A}$ computes the witness not used to give the proofs. This allows to break $G$.

\subsection{Authentication}

We now turn our focus to authentication. Given that we are in the A-KRA model, the sender $S$ knows a secret $s_{S}$ for his public key $p k_{S}$ s.t. $\left(p k_{S}, s_{S}\right) \in R_{S}$ for some poly-time relation $R_{S}$. In the same way, the receiver $R$ knows $s_{R}$ s.t. $\left(p k_{R}, s_{R}\right) \in$ $R_{R}$. Construct a $\Sigma$-protocol $\Sigma=\Sigma_{0} \vee \Sigma_{1}$ for the relation $R=R_{R} \vee R_{S}$, i.e. the verifier $V$ accepts if the prover $P$ knows a secret key for $p k_{S}$ or for $p k_{R}$. Now the parties can identify to each other using this $\Sigma$-protocol.

The way we build an authenticated channel from this identification protocol is as follows: $S$ wants to send $R$ a message $m \in\{0,1\}^{\ell}$, where $\ell$ is a fixed message length. We essentially let the receiver simulate a clock by identifying towards the sender $\ell$ times. In each "time period" the sender will then either identify itself or not. This defines the $\ell$ bits of the message. At the end the sender does a number of identifications to bring up the total number of identifications given by the sender to $\ell$. The receiver will accept only if it sees a total of $\ell$ identifications. This is done to make it impossible for an adversary to drop identifications from the sender to the receiver. At the end, we add two last rounds where $S$ identify to $R$ and then $R$ identifies to $S$. This is to inform the other party that the message was accepted.

For $m \in\{0,1\}^{\ell}$ define $\sigma(m) \in\{\mathrm{R}, \mathrm{S}\}^{2 \ell+2}$ to be $\mathrm{S}^{m_{1}}\|\mathrm{R}\| \mathrm{S}^{m_{2}}\|\mathrm{R}\| \cdots \| \mathrm{S}^{m_{\ell}}$ $\|\mathrm{R}\| \mathrm{S}^{\ell-\sum_{i=1}^{\ell} m_{i}}\|\mathrm{~S}\| \mathrm{R}$, where $m_{i}$ is the $i$-th bit of $m$. Note that $m \neq m^{\prime} \Rightarrow \sigma(m) \neq$ $\sigma\left(m^{\prime}\right)$, that $\sigma(m)$ contains exactly $\ell+1$ symbols of each type, and that the last symbols are always $\mathrm{S} \| \mathrm{R}$. These are sufficient properties for the protocol to be secure. The protocol is given in Fig. 5 .

Theorem 2. If the public keys are set up as in Fig. 3, then the following holds except with negligible probability: If $S$ outputs accept at the end of $\pi_{a u}$ then $R$ outputs (accept, $m$ ), where $m$ was the message input by $S$.

The intuition is as follows: For $I=1, \ldots, 2 \ell+2$ we match the $i$ 'th instance run by $S$ to the $i$ 'th instance run by $R$. If $S$ and $R$ open a prover respectively a verifier, or a verifier respectively a prover, then they might both continue to $I+1$ without rejecting. If $S$ and $R$ both open a verifier, then one of them will be terminated without a prover were running. Therefore this verifier will reject (by Thm. II), which makes the party running that verifier reject. If $S$ and $R$ both instantiate a prover, then one of these provers will close without a verifier having been running at the other party.

Wlog, say that a prover was running at $S$ while no verifier was running at $R$ (one can repeat the argument switching the role of $R$ and $S$ ). This prover will not make any verifier accept at $R$, therefore $S$ will run more provers than the number of accepting verifiers that $R$ runs. Since $S$ starts $\ell+1$ provers, by construction of $\sigma$, it follows that $R$ sees at most $\ell$ accepting verifiers. Therefore $R$ will not output accept. It follows that if $S$ and $R$ have different $\sigma$, then one 
setup: Sender $S$ knows $s_{S}, p k_{R}, R_{S}$ and $R_{R}$ and receiver $R$ knows $p k_{S}, s_{R}, R_{S}$ and $R_{R}$ such that $\left(p k_{S}, s_{S}\right) \in R_{S}$ and $\left(p k_{R}, s_{R}\right) \in R_{R}$.

sender: The first time $S$ gets an input $m \in\{0,1\}^{\ell}$ it computes $\sigma=\sigma(m)$, sends $m$ to $R$ and runs the following:

1. Let $I=1$.

2. If $\sigma_{I}=\mathrm{S}$, then instantiate a prover $P=P\left(\left(p k_{S}, p k_{R}\right), s_{S}\right)$ and let it interact with $R$.

3. If $\sigma_{I}=\mathrm{R}$, then instantiate a verifier $V=V\left(p k_{S}, p k_{R}\right)$ and let it interact with $R$. If $V$ rejects, then terminate the protocol with output reject.

4. When the above instance closes (either $P$ or $V$ ), then let $I=I+1$. If $I \leq 2 \ell+2$, then go to Step 21 If $I>2 \ell+2$, then output accept.

receiver: The first time $R$ receives $m \in\{0,1\}^{\ell}$ from $S$ it computes $\sigma=\sigma(m)$ and runs the following:

1. Let $I=1$.

2. If $\sigma_{I}=\mathrm{R}$, then instantiate a prover $P=P\left(\left(p k_{S}, p k_{R}\right), s_{R}\right)$ and let it interact with $S$.

3. If $\sigma_{I}=\mathrm{S}$, then instantiate a verifier $V=V\left(p k_{S}, p k_{R}\right)$ and let it interact with $S$. If $V$ rejects, then terminate the protocol with output reject.

4. When the above instance closes (either $P$ or $V$ ), then let $I=I+1$. If $I \leq 2 \ell+2$, then go to Step 2 If $I>2 \ell+2$, then output (accept, $m$ ).

Fig. 5. The authentication protocol $\pi_{\mathrm{au}}\left(\left(p k_{S}, p k_{R}\right), s_{S}, s_{R}\right)$

of them does not output accept. In other words, if both parties output accept, then they saw the same message $m$, as $\sigma$ is a unique encoding of $m$.

Second, assume that $R$ did not accept. This implies that $R$ rejected when $I<2 \ell+2$ or at least $R$ never reached $I=2 \ell+2$, as $\sigma_{2 \ell+2}=\mathrm{R}$ implies that $R$ cannot reject while $I=2 \ell+2$. Therefore $R$ ran at most $\ell$ provers and thus $S$ saw at most $\ell$ verifiers accept. Therefore $S$ did not accept either. In other words, if $S$ accepts, then $R$ accepts.

Putting these two observations together, we conclude that if $S$ accepts, then both parties accept, and then $S$ and $R$ saw the same message $m$, as desired. This symmetric guarantee makes the protocol suitable also to authenticate messages from $R$ to $S$, and we will use this property in Thm. [3.

\subsection{Multiparty Authentication}

Our ideal functionality for authenticated transmission is given in Fig. 6. We have it do a key setup as $\mathcal{F}_{\text {KRA }}^{G}$ and output the generated keys before the authenticated transfer phase begins. This is for compositional reasons - it allows outer protocols to use the same secrets, which we exploit in later sections. Here we focus on the phase after the keys are generated: The functionality allows to deliver only messages which were actually sent, which models authentication. It can deliver a message several times and reorder them. This can be handled outside $\mathcal{F}_{\text {MAU }}$ using e.g. sequence numbers. Any message sent is leaked to the adversary to model that the transmission is only authenticated, not private. 
init: First it lets $\operatorname{init}_{i}=1$ for all $P_{i}$, and then it runs $\mathcal{F}_{\text {KRA }}^{G}$ with adversary $\mathcal{A}$, to generate $\left(R_{1}, p k_{1}, s_{1}\right), \ldots,\left(R_{n}, p k_{n}, s_{n}\right)$.

init done: If the adversary inputs (done, $i$ ) at a point where init $_{i}=1$ and after $\mathcal{F}_{\text {KRA }}^{G}$ terminated, then output $\left(p k i, s_{i}\right)$ to $P_{i}$, where $p k i=$ $\left(\left(R_{1}, p k_{1}\right), \ldots,\left(R_{n}, p k_{n}\right)\right)$, and set $\operatorname{init}_{i}=0$.

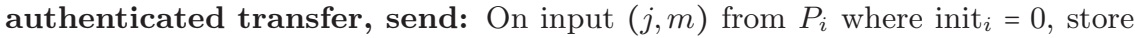
$(i, j, m)$ and output $(i, j, m)$ to the adversary.

authenticated transfer, receive: On input $(i, j, m)$ from the adversary, if $(i, j, m)$ was previously stored, wait until $\operatorname{init}_{j}=0$ and then output $(i, m)$ to $P_{j}$.

Fig. 6. The ideal functionality $\mathcal{F}_{\text {MAU }}^{G}$ for multiparty authenticated communication

Our implementation of $\mathcal{F}_{\mathrm{MAU}}^{G}$ runs in the $\mathcal{F}_{\mathrm{KRA}}^{G}$ hybrid model, see Fig. $\mathbf{7}$

setup: When party $P_{i}$ receives $\left(p k i, s_{i}\right)$ from $\mathcal{F}_{\text {KRA }}^{G}$, it parses $p k i$ as $\left(\left(R_{1}, p k_{1}\right), \ldots,\left(R_{n}, p k_{n}\right)\right)$ and sets init $=1$.

key generation: On its first activation $P_{i}$ generates a random verification key $v k_{i}$ for a digital signature scheme, along with the corresponding signing key $s k_{i}$ and stores (keys, $v k_{i}, s k_{i}$ ). Then $P_{i}$ sends $v k_{i}$ to all other parties.

key authentication: After key generation each ordered pair of parties $\left(P_{i}, P_{j}\right)$ with $i<j$ runs the following in parallel:

- The parties $P_{i}$ and $P_{j}$ run the protocol $\pi_{i, j}=\pi_{\mathrm{au}}\left(\left(p k_{i}, p k_{j}\right), s_{i}, s_{j}\right)$ from Fig. 5

- Party $P_{i}$ uses the input $m=\left(v k_{i}, v k_{j}^{\prime}\right)$, where $v k_{j}^{\prime}$ is the value it received from $P_{j}$ in key generation. Party $P_{j}$ uses the input $m=\left(v k_{i}^{\prime}, v k_{j}\right)$, where $v k_{i}^{\prime}$ is the value it received from $P_{i}$ in key generation.

- If $P_{i}$ accepts in $\pi_{i, j}$, then it stores $\left(\mathrm{vk}, j, v k_{j}^{\prime}\right.$ ). If $P_{j}$ accepts in $\pi_{i, j}$ then it stores (vk, $\left.i, v k_{i}^{\prime}\right)$.

When $P_{i}$ stored (keys, $\left.v k_{i}, s k_{i}\right)$ and (vk, $\left.j, v k_{j}^{\prime}\right)$ for all $P_{j}$ with $j \neq i$, then $P_{i}$ outputs $\left(p k i, s_{i}\right)$ and sets init $=0$.

KRA propagationsthenticated transfer, send: When $P_{i}$ gets input $(j, m)$, where init $=0, P_{i}$ computes $S=\operatorname{sig}_{s k_{i}}(i\|j\| m)$ and sends $(i, j, m, S)$ to $P_{j}$.

authenticated transfer, receive: On a message $(i, j, m, S)$ the party $P_{j}$ waits until init $=0$. Then it looks up $\left(\mathrm{vk}, i, v k_{i}\right)$ and outputs $(i, m)$ if $\operatorname{ver}_{v k_{i}}(i\|j\| m, S)=$ accept.

Fig. 7. The protocol $\pi_{\text {MAU }}^{G}$ for multiparty authenticated communication

Theorem 3. If $G$ is a meaningful key generator, then $\pi_{\mathrm{MAU}}^{G} U C$ securely implements $\mathcal{F}_{\mathrm{MAU}}^{G}$ against a static, active adversary.

The proof is essentially a reduction to Thm. 2] If $\pi_{\mathrm{MAU}}^{G}$ is not secure it is possible to make an honest $P_{j}$ output $(i, m)$ for an honest $P_{i}$ without giving input $(j, m)$ 
to $P_{i}$. We can reduce that to an attack on the protocol in Fig. 5. First of all, we can assume that all other parties than $P_{i}$ and $P_{j}$ are corrupted, as this can only help the adversary. Then, whenever $P_{i}$ or $P_{j}$ have to interact with any $P_{k} \notin\left\{P_{i}, P_{j}\right\}$, they run the protocol honestly, but use the secret $s_{k}$ of $P_{k}$ as witness. By witness indistinguishability (WI) this changes the probability that $P_{j}$ outputs $(i, m)$ without $P_{i}$ having received input $(j, m)$ at most negligibly. But now all interaction involving other parties than $P_{i}$ and $P_{j}$ can be run by the adversary in its head, as it knows $s_{k}$ for all corrupted parties - whatever messages $P_{i}$ would send to $P_{k}$ can be computed using $s_{k}$. But this modified adversary is carrying out an attack on Fig. [7 with $n=2$. This is essentially an attack on Fig. 5. The only difference is that in Fig. [ ] during KRA propagation, the environment gets $s_{i}$ and $s_{j}$ from $P_{i}, P_{j}$. This happens after the protocol $\pi_{i, j}$ was run, and therefore it is not needed to run the adversary against Fig. $\mathbf{5}$

\section{UC-OT in the A-KRA Model given SA-OT}

Suppose we are given an $\mathrm{UC}$ commitment functionality, $\mathcal{F}_{\mathrm{MCOM}}$ as defined in [CF01]: then we can implement UC zero-knowledge, $\mathcal{F}_{\mathrm{MzK}}$, for all NP relations, which in turn allows us to implement a static, active UC secure OT from the passive secure OT. We can therefore focus on implementing $\mathcal{F}_{\text {MCOM }}$ using SA-OT.

The main idea of the protocol in Fig. 8 is to "compile" the SA-OT into a UC-OT using the WI proof for statements of the kind "I followed to protocol or I know your secret key".

Theorem 4. The protocol $\pi_{\mathrm{MCOM}}$ is a UC secure implementation of $\mathcal{F}_{\mathrm{MCOM}}$ in the $\mathcal{F}_{\mathrm{MAU}}^{G}$ hybrid model secure against a static, active adversary.

The simulator extracts a commitment from a corrupted sender $S^{*}$ to an honest receiver $R$ by using selection bit $c=1$ to learn $m$ from the SA-OT. If the sender manages to send $m^{\prime} \neq m$ in the opening phase for some commitment, we can extract the proofs in the SA-OT for this commitment and learn a secret $s_{R}^{\prime}$ for $R$ 's public key $p k_{R}$. Since $R$ never uses $s_{R}$ in the protocol, this contradicts the hardness of computing a witness for $p k_{R}$. To be able to use selection bit $c=1$, the simulator gives the proof in the run of the SA-OT using the secret $s_{S}^{\prime}$ of the sender. This goes unnoticed by the computational hiding of the commitment scheme, the computational hiding of the SA-OT and the WI of the OR-construction. To trapdoor open a commitment to some $m^{\prime}$ the simulator simply sends $m^{\prime}$ and simulates the proof that this is the correct message, by using the secret of the receiver as witness. This goes unnoticed as for $c=1$.

Corollary 1. If there exists a passive secure OT protocol, then any well-formed functionality $\mathcal{F}$ can be UC implemented in the A-KRA model, against a static, active adversary.

Proof: By Thm. 目 we can implement $\mathcal{F}_{\mathrm{MCOM}}$ in the $\mathcal{F}_{\mathrm{MAU}}^{G}$-hybrid model, which implies that we can implement any well-formed $\mathcal{F}$ in the $\mathcal{F}_{\mathrm{MAU}}^{G}$-hybrid model, if there exists a passive secure SA-OT protocol. By Thm. B] we can implement $\mathcal{F}_{\text {MAU }}^{G}$ 
The following describes a commitment to $m$ from party $S$ to party $R$. In the full protocol different instances use session identifiers to separate executions. Here commit $(\cdot)$ is a statistically binding commitment.

1. All communication is authenticated using $\mathcal{F}_{\text {MAU }}^{G}$. Use sequence numbers to guarantee that no identical messages are ever sent, and thus never accept the same message twice from any party.

2. $R$ samples a uniformly random string $u$ and sends $U \leftarrow \operatorname{commit}(u)$ to $S$.

3. $S$ samples a uniformly random string $v$, sets $m_{0}=1^{|m|}$, sets $m_{1}=m$ and sends $V \leftarrow \operatorname{commit}(v), M_{0} \leftarrow \operatorname{commit}\left(m_{0}\right)$ and $M_{1} \leftarrow \operatorname{commit}\left(m_{1}\right)$ to $R$.

4. Then $S$ and $R$ run the SA-OT, where $S$ takes inputs $m_{0}$ and $m_{1}$ and uses randomness $v$ while $R$ gives input $c=0$ and uses randomness $u$. After sending each message in the SA-OT $R$ shows that it knows an opening of $U$ to $u$ such that the message it sent is consistent with having run the SA-OT with randomness $u$, selection bit $c=0$ and the messages received from $R$ so far. After sending each message in the SA-OT $S$ shows that it knows an opening of $V, M_{0}$ and $M_{1}$ to $v, m_{0}$ respectively $m_{1}$ such that the messages it sent are consistent with the execution of the SA-OT with randomness $v$, inputs $m_{0}$, $m_{1}$ and the messages received from $S$. The proofs are given via a $\Sigma$-protocol for NP and use the OR-construction to prove either knowledge of the openings mentioned above or the secret of the other party.

5. To open $S$ sends $m$ to $R$ and shows that $m$ is the message inside $M_{1}$ or that $S$ knows $s_{R}$ such that $\left(p k_{R}, s_{R}\right) \in R_{R}$. The proof is given using two $\Sigma$-protocols and the OR-construction.

Fig. 8. The protocol $\pi_{\mathrm{Mcom}}$ for UC commitments using SA-OT

in the unauthenticated $\mathcal{F}_{\mathrm{KRA}}^{G}$-hybrid model for any meaningful $G$. It then follows from the UC composition theorem that we can implement any well-formed $\mathcal{F}$ in the unauthenticated $\mathcal{F}_{\mathrm{KRA}}^{G}$-hybrid model for any meaningful $G$, if there exists a passive secure SA-OT protocol.

\section{UC Commitment in the C-KRA Model given OWFs}

In a nutshell, to construct UC-Com in the C-KRA model, we let the public keys to be commitments of the secret keys. Then to commit the sender send an encryption of the message under his secret key. To open, he sends the message $m$ together with a WI proof for a statement " $m$ is the committed message or I know your secret key".

Theorem 5. If one-way functions exist, then there exists a UC commitment scheme for the C-KRA model secure against a static, active adversary.

Proof: The public key is an unconditionally binding commitment $p k_{i}=$ $\operatorname{commit}\left(K_{i} ; r_{i}\right)$ to a uniformly random value $K_{i} \in_{R}\{0,1\}^{\kappa}$. Let $F_{\{0,1\}^{\kappa}}$ : $\{0,1\}^{2 \kappa} \rightarrow\{0,1\}^{2 \kappa}$ be a pseudo-random permutation (PRP). Both can be instantiated using one-way functions. 
To commit to $m \in\{0,1\}^{\kappa}$ with session identifier sid $\in\{0,1\}^{\kappa}$ towards $P_{j}, P_{i}$ sends $M=F_{K_{i}}(\operatorname{sid} \| m)$. To open the commitment to $P_{j}$, the sender sends $m$ and gives a proof that it knows $K$ and $r$ such that " $p k_{j}=\operatorname{commit}(K ; r) \vee\left(p k_{i}=\right.$ $\left.\operatorname{commit}(K ; r) \wedge M=F_{K}(\operatorname{sid} \| m)\right)$ ". The proof is given using two $\Sigma$-protocols combined with the OR-construction.

To extract, the simulator computes $m=F_{K_{i}}^{-1}(M)$, where $K_{i}$ is found as part of the secret $s_{i}=\left(K_{i}, r_{i}\right)$ of the sender $P_{i}$. By $p k_{i}$ binding the sender to $K_{i}$ unconditionally and the soundness of the proof and the fact that the sender cannot open the commitment $p k_{j}$, this will yield the only $m$ that the sender can open the commitment to later.

To equivocate the simulator sends a uniformly random $M$. When given $m$ it sends $m$ and gives the proof using the secret $s_{j}$ of the receiver as witness. By computational hiding of the commitment scheme, pseudo-randomness of $F$ and WI of the proof, this will go unnoticed.

\section{UC OT in the A-CRS Model given SA-OT}

Here we implement UC OT from SA-OT in any CRS model. We prove it for the A-CRS model, and hence for the U-CRS and C-CRS models too. We already know how to do UC OT in the A-KRA model given SA-OT, so it is sufficient to implement $\mathcal{F}_{\mathrm{KRA}}^{G}$ in the $\mathcal{F}_{\mathrm{CRS}}^{D}$ for any meaningful $G$ and all one-way $D$.

Theorem 6. If $D$ is $O W F$, then $G^{D}$ is meaningful, and if the used OT protocol is a SA-OT, then $\pi_{\mathrm{KRA}}^{D}$ in Fig. 9 is a UC secure implementation of $\mathcal{F}_{\mathrm{KRA}}^{G^{D}}$ in the $\mathcal{F}_{\mathrm{CRS}}^{D}$-hybrid model against a static, active adversary.

The protocol runs in the $\mathcal{F}_{\mathrm{CRS}}^{D}$-hybrid model.

- All parties $P_{i}$ receive $(D, c r s)$ from $\mathcal{F}_{\mathrm{CRS}}^{D}$.

- Each $P_{i}$ samples $p k_{i}=D\left(s_{i}\right)$ for a uniformly random $s_{i}$ and sends $p k_{i}$ to all parties. All parties resend the received value $p k_{i}$ to all parties.

- Then in round-robin, for $i=1, \ldots, n$, each $P_{i}$ proves knowledge of $s_{i}$ to all other parties. It does this in round robin, for $j=1, \ldots, n$. With each $P_{j}$ it runs the proof as in Fig. [8] It inputs $m_{0}=0^{\left|s_{i}\right|}$ and $m_{1}=s_{i}$ to the SA-OT and $P_{j}$ inputs $c=0$. During the run of the SA-OT, $P_{i}$ proves that either 1) its messages are consistent with a run of the SA-OT protocol and $p k_{i}=D\left(m_{1}\right)$ or 2) its messages are consistent with a run of the SA-OT protocol and $\mathrm{crs}=$ $D\left(m_{1}\right)$. Party $P_{j}$ proves that either 1$)$ its messages are consistent with a run of the SA-OT protocol with $c=0$ or 2 ) it knows $s$ such that $\operatorname{cr} s=D(s)$. The proofs are given via a $\Sigma$-protocol for NP and the OR-construction. When $P_{i}$ and $P_{j}$ are done, they both send done to the other parties. Parties only begin their proof when they received done from all previous pairs.

- If and when a party $P_{k}$ received $c r s$ from $\mathcal{F}_{\text {CRS }}^{D}$, a value $p k_{i}$ from each $P_{i}$ and a resent value $p k_{i}^{\prime}$ from all other parties $P_{j}$ with $p k_{i}^{\prime}=p k_{i}$, and saw an accepting proof from each $P_{i}$, it outputs $\left(\left(p k_{1}, R^{D}\right), \ldots,\left(p k_{n}, R^{D}\right)\right), s_{k}$.

Fig. 9. The protocol $\pi_{\text {KRA }}^{D}$ that implements a KRA in the A-CRS model 
The proof is very similar to the proof of Thm. 4 The simulator extracts the secret of corrupted parties using selection bit $c=1$. It simulates proofs using the secret $s$ of $\mathrm{crs}$. We run the proofs in round-robin to ensure that the simulator will not give a simulated proof (using $s$ ) while a corrupted party has to give a proof. If it did so, we could not show that a corrupted party cannot give an acceptable proof unless it used $m_{1}$ such that $p k_{i}=D\left(m_{1}\right)$ in the SA-OT. When the proofs are run in round-robin, we can.

\section{UC-Com in the A-KRA Model Implies SA-OT}

\section{Theorem 7. SA-OT is necessary for UC-Com in the A-KRA model.}

Proof: We show how a UC secure commitment scheme for the A-KRA model can be turned into a SA-OT. Note that this UC-Com protocol needs to work for any KRA, so we can choose a special KRA that it's possible to "simulate" in some sense without using any setup assumptions.

To simplify the proof, consider the AND primitive, where $A$ inputs $a \in\{0,1\}$ and $B$ inputs a bit $b \in\{0,1\}$ and where $A$ has no output and $B$ gets output $c=a b$. It is well-known that if there exists passive, stand-alone secure AND (SA-AND), then there also exist SA-OT. Then it is sufficient to show how to implement SA-AND from UC-Com in the A-KRA model.

The existence of UC-Com clearly implies OWFs, so we can assume that we have a PRG $g:\{0,1\}^{\kappa} \rightarrow\{0,1\}^{\kappa+1}$. Consider the key generator $G^{f}$, where $f$ : $\{0,1\} \times\{0,1\}^{\kappa} \times\{0,1\}^{\kappa+1} \rightarrow\{0,1\}^{\kappa+1} \times\{0,1\}^{\kappa+1}$ and $f\left(b, r^{b}, p k^{1-b}\right)=\left(p k^{0}, p k^{1}\right)$ for $p k^{b}=g\left(r^{b}\right)$. This is clearly a meaningful generator, as a PRG $g:\{0,1\}^{\kappa} \rightarrow$ $\{0,1\}^{\kappa+1}$ is one-way.

From the assumption that there exist UC-Com in the A-KRA model, we have a protocol $\pi$ which UC implements $\mathcal{F}_{\text {MCOM }}$ in the $\mathcal{F}_{\text {KRA }}^{G}$-hybrid model with sender $S$ and receiver $R$. The sender gets key material $\left(p k_{S}, s_{S}\right)$ and $p k_{R}$ and the receiver gets key material $p k_{S}$ and $\left(p k_{R}, s_{R}\right)$. Here $p k_{i}=f\left(s_{i}\right)$ for $i=S, R$.

Consider the following adversary $\mathcal{A}$ against $\pi$ for the case when the sender is corrupted: It samples uniformly random $c \in\{0,1\}, r_{S}^{c} \in\{0,1\}^{\kappa}$ and $r_{S}^{1-c} \in$ $\{0,1\}^{\kappa}$ and lets $p k_{S}^{c}=g\left(r_{S}^{c}\right)$ and $p k_{S}^{1-c}=g\left(r_{S}^{1-c}\right)$. Then it inputs $s_{S}^{\prime}=(1-$ $\left.c, r_{S}^{1-c}, p k_{S}^{c}\right)$ to $\mathcal{F}_{\mathrm{KRA}}^{G^{f}}$. Then it commits to some $m \in\{0,1\}$ by honestly running the commitment phase of the protocol $\pi$ with key material $\left(p k_{S}, s_{S}\right)$ and $p k_{R}$, where $s_{S}=\left(c, r_{S}^{c}, p k_{S}^{1-c}\right)$. It's clear here that $s_{S} \neq s_{S}^{\prime}$ as the first bit is different, and $f\left(s_{S}\right)=f\left(s_{S}^{\prime}\right)$. Later it decommits by honestly running the opening phase of the protocol $\pi$.

Lemma 1. When running with $\mathcal{A}$, the honest receiver $R$ will accept the commitment and will later accept the opening to $m$, except with negligible probability.

The proof follows from the fact that $R$ cannot distinguish $\mathcal{A}$ from the honest sender $S$. By $\pi$ being UC secure, and the above lemma, it follows that there exists a UC simulator $\mathcal{S}$ which can extract $m$ from the conversation with $\mathcal{A}$ already in the commitment phase. Since $\mathcal{S}$ is simulating $\mathcal{F}_{\text {KRA }}^{G^{f}}$ to $\mathcal{A}$, it follows that $\mathcal{S}$ learns $s_{S}^{\prime}$ and chooses the value of $p k_{R}$. 
1. First $B$ samples $c \in\{0,1\}$ uniformly at random. Then, if $b=1$, it uses $\mathcal{S}$ to sample $p k_{R}$, samples $r_{S}^{1-c} \in\{0,1\}^{\kappa}$ uniformly at random and lets $p k_{S}^{1-c}=$ $g\left(r_{S}^{1-c}\right)$. If $b=0$, then $B$ samples $p k_{R}=f\left(s_{R}\right)$ for uniformly random $s_{R}$ and samples uniformly random $p k_{S}^{1-c} \in_{R}\{0,1\}^{\kappa+1}$. In both cases it sends $\left(c, p k_{S}^{1-c}\right)$ and $p k_{R}$ to $A$.

At the same time $A$ samples uniformly random $r_{S}^{c} \in_{R}\{0,1\}^{\kappa}$, lets $p k_{S}^{c}=g\left(r_{S}^{c}\right)$ and sends $p k_{S}^{c}$ to $B$.

2. Both parties let $p k_{S}=\left(p k_{0}^{S}, p k_{1}^{S}\right)$. $A$ sets $s_{S}=\left(c, r_{S}^{c}, p k_{S}^{1-c}\right)$. If $b=1$ then $B$ lets $s_{S}^{\prime}=\left(1-c, r_{S}^{1-c}, p k_{S}^{c}\right)$. Note that in this case $f\left(s_{S}\right)=p k_{S}=f\left(s_{S}^{\prime}\right)$.

3. A inputs $a$ by committing to $m=a$ by honestly running the commitment phase of $\pi$, playing the role of the sender $S$ with key material $\left(p k_{S}, s_{S}\right)$ and $p k_{R}$.

If $b=1$, then $B$ runs $\mathcal{S}$ to extract $a$ from the conversation with $A$, and outputs $a$. If $b=0$, then $B$ honestly runs the commitment phase of $\pi$, playing the role of the receiver $R$ with key material $\left(p k_{R}, s_{R}\right)$ and $p k_{S}$, and outputs 0 .

Fig. 10. SA-AND protocol

Consider then the SA-AND in Fig. [10, If $b=1$, then all values are distributed as in the simulation of $\pi$ with $\mathcal{A}$ and $\mathcal{S}$, so $B$ computes $a$, except with negligible probability. This established the correctness, hence it only remains to prove the following lemma.

Lemma 2. 1) When $A$ and $B$ are honest, then the view of $A$ when $b=0$ and $b=1$ are computationally indistinguishable. 2) When $A$ and $B$ are honest and $b=0$, then the views of $B$ when $a=0$ and $a=1$ are computationally indistinguishable.

Part 1) follows readily from the fact that by UC security $R$ and $\mathcal{S}$ cannot be distinguished by $\mathcal{A}$. Part 2) follows readily from the fact that a commitment hides the message when both parties are honest.

\section{Conclusions}

Combining our findings with some previous results it is possible to fill the rows of Table 1. We will make use of the following:

Theorem 8. [IR89] There is no black-box construction of SA-OT from OWF.

Theorem 9. [R89] There is no black-box construction of key-agreement (KA) from $O W F$.

Theorem 10. DG03] UC-Com in the U/A-CRS model implies SA-OT.

Theorem 11. [DG03] UC-Com in the C-CRS model implies KA.

The answer to (a) follows directly from Thm. 11] and Thm. 9] for the CRS models; in the same way it follows from (j) and Thm. 8 for the A-KRA model; (b) is shown in Thm. 15. (c) follows from Thm.9] and the fact that UC-OT in any model 
Table 1. Questions and answers: If a cell contains more than one element it means that the answer, $\mathrm{Y}(\mathrm{es})$ or $\mathrm{N}(\mathrm{o})$, in the row is true for all elements in the cell. As an example, row (g) says that the answer to the question is SA-OT sufficient for UC-OT in the A-CRS model? is yes.

\begin{tabular}{|c|c|c|c||c||c|}
\hline & Assumption & & Functionality & Model & Answer \\
\hline \hline (a) & OWF & suf. & UC-Com & U/C/A-CRS, A-KRA & N \\
\hline (b) & OWF & suf. & UC-Com & C-KRA & Y \\
\hline (c) & OWF & suf. & UC-OT & U/C/A-CRS, C/A-KRA & N \\
\hline \hline (d) & UC-Com & suf. & UC-OT & U/A-CRS, A-KRA & Y \\
\hline (e) & UC-Com & suf. & UC-OT & C-CRS & open \\
\hline (f) & UC-Com & suf. & UC-OT & C-KRA & N \\
\hline \hline (g) & SA-OT & suf. & UC-Com, UC-OT & U/C/A-CRS, C/A-KRA & Y \\
\hline \hline (h) & OWF & nec. & UC-Com, UC-OT & U/C/A-CRS, C/A-KRA & Y \\
\hline \hline (i) & UC-Com & nec. & UC-Com, UC-OT & U/C/A-CRS, C/A-KRA & Y \\
\hline \hline (j) & SA-OT & nec. & UC-Com & U/A-CRS, A-KRA & Y \\
\hline (k) & SA-OT & nec. & UC-Com & C-CRS & open \\
\hline (l) & SA-OT & nec. & UC-Com & C-KRA & N \\
\hline (m) & SA-OT & nec. & UC-OT & U/A/C-CRS, A-KRA & Y \\
\hline (n) & SA-OT & nec. & UC-OT & C-CRS & open \\
\hline
\end{tabular}

implies KA; the answer to (d) is built from the fact that UC-Com in those models implies SA-OT (see (j)), and that we can compile this into a UC-OT using the UC-Com, as it implies UC-ZK; the answer to (f) goes as follows: (m) tells us that UC-OT in the C-KRA model implies SA-OT while (b) tells us that OWF are sufficient for UC-OT in the C-KRA model. Therefore UC-Com is not sufficient for UC-OT, or we will get a contradiction with Thm. 8 (g) is proved in Thm. 4 and [6. (h) is trivial as OWF are minimal for cryptography, and (i) is trivial as UC-OT is complete for UC computation; ( $\mathrm{j}$ ) is proved in Thm. 7 and [0] (1) follows from (b) and Thm. 8 ; finally $(\mathrm{m})$ follows from the following observation: semi-honest parties can efficiently simulate the U-CRS (or the A-CRS) setup model by letting one party pick a random string without learning the trapdoor and make the crs public. Then the parties will run the UC-OT protocol using this string as the CRS, therefore achieving a SA-OT. As for the C-KRA (or the A-KRA) models, they can be efficiently simulated by letting every party generate his own public/secret key pair and sending the public key to all other parties. Now the parties can run the UC-OT using those public keys, and they'll achieve a SA-OT.

\subsection{The C-CRS Setup Assumption}

In this section we discuss the C-CRS model, and the open questions (e), (k) and (n) left in the table. Consider ( $\mathrm{n})$ : is SA-OT necessary for UC-OT in the C-CRS model? The way we positively answered the question for the other setup models is by letting one party honestly pick a random CRS and publish it, therefore 
simulating the setup model. We don't know how to do it in the C-CRS model: in fact, we don't know whether it is possible, for any chosen OWF $f$, to sample an image $y=f(x)$ without learning the pre-image $x$. For instance, if $x \in \mathbb{Z}_{q}$ and $f(x)=\left(g^{x}, h^{x}\right)$ for $g, h$ elements in group of large prime order $q$, then it is believed that one cannot sample from the image of $f$ without learning $x$, to the extent that people construct protocols based on this belief (the so-called knowledge of exponent assumption Dam91). This suggests very strongly that the open questions cannot be solved using the techniques we have used here. It could of course be possible to approach (n) in some other way. It seems counterintuitive to think that it would be possible to implement UC-OT in a world where SA-OT does not exist: how much power does a symmetric setup as the $\mathrm{C}-\mathrm{CRS}$ give to the parties? However, if it turns out that the answer to $(\mathrm{n})$ is affirmative, then we could use (g) to turn any UC-OT in the C-CRS model into a UC-OT in the U/A-CRS model, and this would also be a surprising result. Similar considerations can be made for (e) and $(\mathrm{k})$.

Acknowledgements. We would like to thank Yuval Ishai and the anonymous reviewers for many valuable comments.

\section{References}

[BCNP04] Barak, B., Canetti, R., Nielsen, J.B., Pass, R.: Universally composable protocols with relaxed set-up assumptions. In: FOCS, pp. 186-195 (2004)

[Can01] Canetti, R.: Universally composable security: A new paradigm for cryptographic protocols. In: FOCS, pp. 136-145 (2001)

[CDS94] Cramer, R., Damgård, I., Schoenmakers, B.: Proofs of partial knowledge and simplified design of witness hiding protocols. In: Desmedt, Y.G. (ed.) CRYPTO 1994. LNCS, vol. 839, pp. 174-187. Springer, Heidelberg (1994)

[CF01] Canetti, R., Fischlin, M.: Universally composable commitments. In: Kilian, J. (ed.) CRYPTO 2001. LNCS, vol. 2139, pp. 19-40. Springer, Heidelberg (2001)

[CGGM00] Canetti, R., Goldreich, O., Goldwasser, S., Micali, S.: Resettable zeroknowledge (extended abstract). In: STOC, pp. 235-244 (2000)

[CLOS02] Canetti, R., Lindell, Y., Ostrovsky, R., Sahai, A.: Universally composable two-party and multi-party secure computation. In: STOC, pp. 494-503 (2002)

[Dam91] Damgård, I.: Towards practical public key systems secure against chosen ciphertext attacks. In: Feigenbaum, J. (ed.) CRYPTO 1991. LNCS, vol. 576, pp. 445-456. Springer, Heidelberg (1992)

[DG03] Damgård, I., Groth, J.: Non-interactive and reusable non-malleable commitment schemes. In: STOC, pp. 426-437 (2003)

[IPS08] Ishai, Y., Prabhakaran, M., Sahai, A.: Founding cryptography on oblivious transfer - efficiently. In: Wagner, D. (ed.) CRYPTO 2008. LNCS, vol. 5157, pp. 572-591. Springer, Heidelberg (2008)

[IR89] Impagliazzo, R., Rudich, S.: Limits on the provable consequences of oneway permutations. In: STOC, pp. 44-61 (1989)

[Kil88] Kilian, J.: Founding cryptography on oblivious transfer. In: STOC, pp. 20-31 (1988) 
[KL07] Kidron, D., Lindell, Y.: Impossibility results for universal composability in public-key models and with fixed inputs. Cryptology ePrint Archive, Report 2007/478 (2007)

[LPV09] Lin, H., Pass, R., Venkitasubramaniam, M.: A unified framework for concurrent security: universal composability from stand-alone nonmalleability. In: STOC, pp. 179-188 (2009)

[MPR09] Maji, H.K., Prabhakaran, M., Rosulek, M.: A zero-one law for deterministic 2-party secure computation (2009) (manuscript)

[OPV08] Ostrovsky, R., Persiano, G., Visconti, I.: Constant-round concurrent non-malleable zero knowledge in the bare public-key model. In: Aceto, L., Damgård, I., Goldberg, L.A., Halldórsson, M.M., Ingólfsdóttir, A., Walukiewicz, I. (eds.) ICALP 2008, Part II. LNCS, vol. 5126, pp. 548559. Springer, Heidelberg (2008)

[PR08] Prabhakaran, M., Rosulek, M.: Cryptographic complexity of multi-party computation problems: Classifications and separations. In: Wagner, D. (ed.) CRYPTO 2008. LNCS, vol. 5157, pp. 262-279. Springer, Heidelberg (2008)

[RTV04] Reingold, O., Trevisan, L., Vadhan, S.P.: Notions of reducibility between cryptographic primitives. In: Naor, M. (ed.) TCC 2004. LNCS, vol. 2951, pp. 1-20. Springer, Heidelberg (2004) 